\title{
COMPARAÇÃO DE TÉCNICAS NUMÉRICAS PARA OBTENÇÃo DE DISTRIBUIÇÃO DE TAMANHO DE GOTAS EM EMULSÕES UTILIZANDO DADOS ESPECTROSCÓPICOS
}

\author{
C. F. B. SILVA ${ }^{1 *}$, C. ASSENHAIMER ${ }^{1}$, R. GUARDANI ${ }^{1}$ \\ ${ }^{1}$ Universidade São Paulo, Escola Politécnica, Departamento de Engenharia Química \\ *e-mail: carlosfbueno@ usp.br
}

\begin{abstract}
RESUMO
Este trabalho tem como objetivo a implementação e comparação de técnicas para resolução de problemas de inversão, desenvolvendo algoritmos que forneçam distribuições de tamanho de partículas em dispersões a partir de dados de espectroscopia UV-Vis. Foram implementadas quatro técnicas, sendo uma delas um método alternativo sem uso de um método de inversão. Os métodos que utilizaram algum algoritmo de inversão evidenciaram a dificuldade em se obterem distribuições de tamanho de gotas (DTG) de boa qualidade, enquanto o método alternativo foi aquele que se mostrou mais eficiente e confiável.
\end{abstract}

\section{INTRODUÇÃO}

O desenvolvimento de algoritmos computacionais para a obtenção de distribuição de tamanhos de partículas em dispersões e que utilizam dados espectroscópicos em tempo real e "in-line" a partir de sensores, permitirá uma variedade de aplicações, que vão desde o acompanhamento de processos de polimerização, ao tratamento de efluentes e sensoriamento atmosférico.

$\mathrm{O}$ presente estudo visa o desenvolvimento de algoritmos para a obtenção de distribuições de tamanhos de partículas a partir de dados de espectroscopia UV-Vis. Esse processo é caracterizado como um problema inverso, em que são utilizados dados reais de medições de parâmetros observáveis para inferir valores dos parâmetros de um modelo (TARANTOLA, 2004). No caso da espectroscopia, o problema inverso se dá pela análise adequada do campo de espalhamento para descrever as partículas que são responsáveis por esse espalhamento (BOHREN; HUFFMAN, 1983).

Segundo Bohren e Huffman (1983), as informações necessárias para especificar uma partícula são os vetores amplitude e fase do campo espalhado em todas as direções e o campo dentro da partícula. O campo dentro da partícula não é usualmente medido de maneira direta, porém, sob certas condições, ele pode ser aproximado pelo campo incidente. A amplitude e a fase do campo espalhado não são impossíveis de serem obtidos em princípio, porém são raramente determinados na prática. A medida geralmente disponível para análise é a irradiância do campo espalhado em várias direções. Tem-se, portanto, a tarefa de tentar descrever uma partícula (ou uma população de partículas) com um conjunto de dados menor do que a quantidade teoricamente ideal. Com tais condições adversas, só é possível descrever tais partículas utilizando informações 
suplementares obtidas através de modelos ópticos de espalhamento de luz adequados.

Portanto, para realizar o processo de inversão de dados, a fim de obter uma distribuição de tamanho de partículas a partir de dados espectroscópicos é necessária a escolha prévia de um modelo óptico de espalhamento adequado e uma técnica eficiente de inversão.

\section{REVISÃO BIBLIOGRÁFICA}

Neste tópico serão descritos os modelos de espalhamento e algoritmos de inversão, além de um algoritmo alternativo, que não utiliza técnicas de inversão.

\subsection{Modelos ópticos de espalhamento}

A Figura 1 ilustra os diversos processos que ocorrem quando um feixe de luz com comprimento de onda $\lambda_{0}$ incide em uma partícula.

Figura 1 - Mecanismos de interação entre a radiação incidente e uma partícula

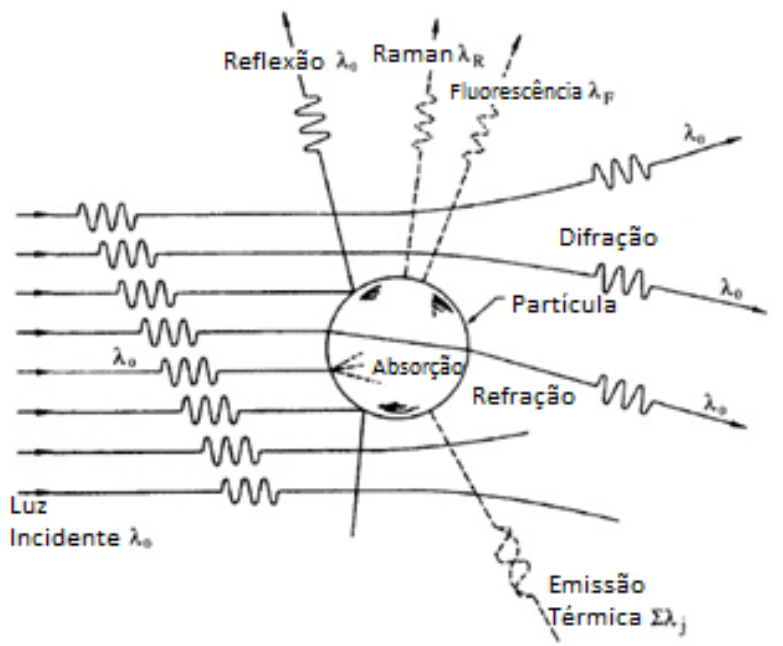

Fonte: Seinfeld e Pandis (2006)

Comumente é dito que a presença das partículas resulta na extinção do raio incidente. Se o meio em que as partículas estão contidas não absorve energia, a diferença das energias incidente e a captada após a interação luz-partícula é contabilizada como absorção (portanto, trata-se da transformação da energia eletromagnética em outras formas) e pelo espalhamento. A extinção nada mais é do que a combinação desses dois fenômenos:

$$
\text { Extinção = Absorção }+ \text { Espalhamento }
$$

É importante ressaltar que a extinção depende da composição química das partículas, de seus tamanhos, forma, orientação, do meio que as envolve, do número de partículas e do estado de polarização e frequência do feixe incidente.

Entre os fenômenos que compõem a extinção, o espalhamento é o responsável por fornecer informações para a estimativa da DTG (FRIEDLANDER, 2000). O tipo de espalhamento será considerado elástico (quando o comprimento de onda da luz espalhada é o mesmo da luz incidente) para cálculos posteriores. Dentre os modelos ópticos contidos dentro do espalhamento elástico, estão os modelos de espalhamento Rayleigh, Mie e Geométrico.

Figura 2 - Padrão dos espalhamentos Rayleigh, Mie e Geométrico

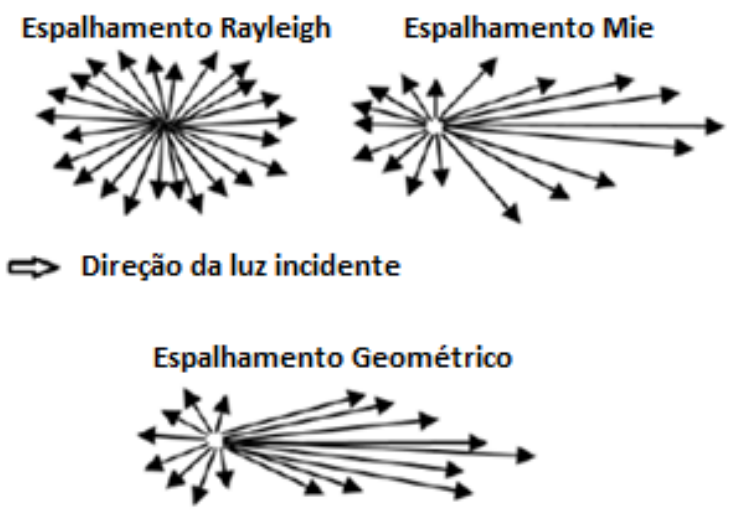

Fonte: Khatib (2014)

A utilização de um modelo em detrimento aos outros (Figura 2) é definida de acordo com a relação entre o tamanho das partículas e o comprimento de onda da luz incidente: 
$\alpha=\frac{2 \pi r}{\lambda}$

De acordo com o parâmetro adimensional $\alpha$, define-se qual modelo óptico mais adequado para caracterizar $o$ espalhamento de luz (SEINFELD; PANDIS, 2006).

$\alpha \ll 1-$ Espalhamento Rayleigh (quando a partícula é pequena comparada ao comprimento de onda)

$\alpha \simeq 1$ - Espalhamento Mie (quando a partícula e o comprimento de onda têm tamanhos semelhantes)

$\alpha \gg 1$ - Espalhamento Geométrico (quando a partícula é grande comparada ao comprimento de onda)

Neste trabalho, as partículas possuem tamanhos com ordem de grandeza semelhante aos comprimentos de onda de luz incidente. Dessa forma, o parâmetro $\alpha$ é próximo de 1 . Logo, foi adotado o modelo de Mie. Van de Hulst (1981) explicita detalhadamente todos os cálculos adotados por este modelo e Mätzler (2002a, 2002b) apresenta alguns scripts em MATLAB ${ }^{\circledR}$ contendo tais cálculos.

Segundo o modelo de Mie, é possível relacionar a turbidez de uma amostra com a sua distribuição de tamanho de partículas através da eficiência de extinção:

$\tau(\lambda)=\frac{\pi}{4} \int_{0}^{\infty} Q_{\text {ext }}\left(\lambda, D_{p}\right) D_{p}^{2} f\left(D_{p}\right) d D_{p}$

$\mathrm{Na}$ Equação 2, os valores de turbidez $(\tau)$ e respectivos comprimentos de onda $(\lambda)$ são conhecidos. O termo $Q_{\text {ext }}$ corresponde à eficiência de extinção e pode ser determinado pelo Modelo de Mie. O termo desconhecido na equação é, portanto, a função de distribuição de gotas $f\left(D_{p}\right)$. A Equação 2 pode ainda ser simplificada por um processo de discretização via quadratura (TWOMEY, 1977). Tem-se:
$\tau=A f$

O termo $A$ corresponde a uma matriz que contém as informações ópticas, cuja inversa é difícil de ser determinada por ser uma matriz quase singular. Dessa maneira, é necessária a utilização de algoritmos de inversão apropriados, os quais serão destacados a seguir.

\subsection{Algoritmos testados}

Abaixo, os algoritmos numéricos utilizados para resolver o problema de inversão são descritos brevemente.

\subsubsection{Método de Veselovskii}

Veselovskii et al. (2002) propõe utilizar uma técnica baseada em ponderação de triângulos para resolver o problema de inversão da matriz $A$ presente na Equação 2.

Pode-se adotar a seguinte expressão:

$K\left(\lambda, D_{p}\right)=\frac{\pi}{4} Q_{\text {ext }}\left(\lambda, D_{p}\right) D_{p}^{2}$

Substituindo a Equação 4 na Equação 2, tem-se:

$\tau(\lambda)=\int_{0}^{\infty} K\left(\lambda, D_{p}\right) f\left(D_{p}\right) d D_{p}$

Reescrevendo a Equação 5, através de uma somatória que consiste na superposição das funções $B_{j}\left(D_{p}\right)$, obtém-se:

$$
f\left(D_{p}\right)=\sum_{j} C_{j} B_{j}\left(D_{p}\right)+\varepsilon
$$

$\mathrm{Na}$ expressão acima, o termo $C_{j}$ corresponde a um vetor de pesos e $B_{j}\left(D_{p}\right)$ é uma função discretizada (vetor) do tipo Bspline, assumindo uma forma triangular. 


$$
B_{j}(D)=\left\{\begin{array}{cc}
0 ; & D \leq D_{j-1} \\
1-\frac{D_{j}-D}{D_{j}-D_{j-1}} ; & D_{j-1}<D \leq D_{j} \\
1-\frac{D-D_{j}}{D_{j+1}-D_{j}} ; & D_{j}<D \leq D_{j+1} \\
0 ; & D>D_{j+1}
\end{array}\right.
$$

O termo $C_{j}$ é calculado através da seguinte expressão:

$C=\left(A^{T} A+\gamma H\right)^{-1} A^{T} \tau$

O termo $H$ corresponde a uma matriz de suavização. A construção dessa matriz é mostrada por Twomey (1977). O termo $\gamma$ é chamado de parâmetro de regularização e seu cálculo é feito utilizando a técnica Generalized Cross Validation (GCV) detalhada por Green e Silverman (1994). O termo A, por sua vez, é dado pela integral:

$A=\int_{D_{\min }}^{\nu_{\max }} K\left(\lambda, D_{p}\right) B_{j}\left(D_{p}\right) d D_{p}$

Sendo $K\left(\lambda, D_{p}\right)$ uma função Kernel.

Tendo definidos $B_{j}\left(D_{p}\right)$ e $C_{j}$, basta utilizar a equação para determinar a DTG. A Figura 3 ilustra a ponderação dos triângulos para se obter uma curva de distribuição.

Figura 3 - Ponderação de triângulos: $\boldsymbol{C}_{\boldsymbol{j}}$ representa o peso (altura) e $x$ a base de cada triângulo

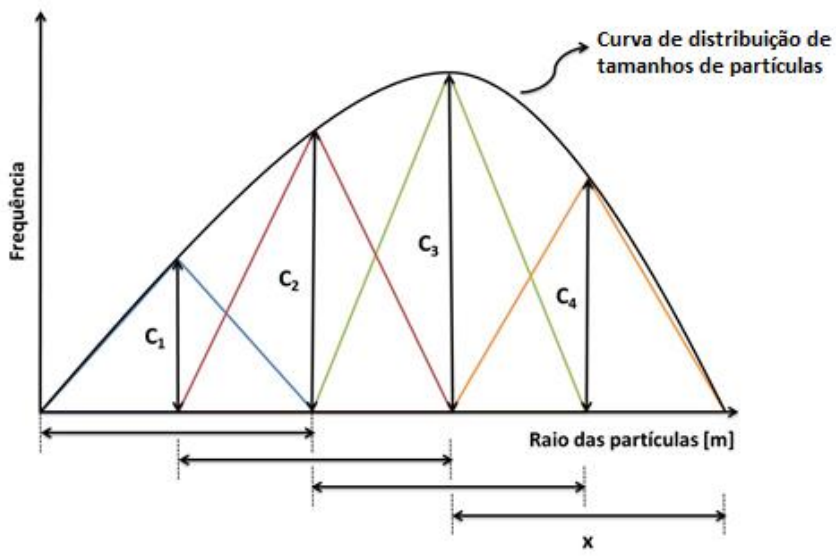

2.2.2 Non-Negative Least Squares (NNLS)
A técnica NNLS é uma das alternativas para solucionar o problema de inversão da matriz $A$. Trata-se de uma versão da técnica de minimização quadrática, com a inserção de uma restrição de não-negatividade nos componentes da função de distribuição final. Matematicamente, tem-se:

$\min _{f}\|A f-\tau\|_{2}$ tal que $f \geq 0$

As propriedades ópticas necessárias para a construção da matriz A estão baseadas na teoria de Mie e foram calculadas a partir dos scripts elaborados por Mätzler (2002a, 2002b). Foi utilizada a rotina lsqnonneg contida na biblioteca de funções do MATLAB ${ }^{\circledR}$ para a resolução do problema de inversão desta matriz. O detalhamento da técnica é apresentado por Lawson e Hanson (1995).

\subsubsection{Regularização Phillips-Twomey (PTR)}

A técnica PTR também tem como função a resolução de problemas de inversão de matrizes quase singulares. Ela propõe que a Equação 2 seja reescrita, inserindo-se alguns parâmetros auxiliares. A principal característica dessa técnica está na suavização da resposta final.

$f=\left(A^{T} A+\gamma H\right)^{-1} A^{T} \tau$

A construção da matriz A é demonstrada por Eliçabe e Garcia-Rubio (1990). Os termos $\gamma$ (parâmetro de regularização) e $H$ (matriz responsável pela suavização da curva f) são obtidos como descritos no Método Veselovskii.

Os cálculos das propriedades ópticas realizados pelos algoritmos estão baseados no modelo de Mie e os scripts auxiliares são detalhados por Mätzler (2002a, 2002b).

\subsubsection{Método de Busca Direta (MBD)}

Este método propõe uma solução alternativa às técnicas de inversão. $\mathrm{O}$ princípio deste algoritmo consiste em 
comparar um espectro de extinção de uma amostra com vários espectros-teste. Através de uma busca utilizando a minimização quadrática entre a amostra e os testes, obtémse o espectro teste mais próximo da amostra. A DTG correspondente a este espectro-teste deverá ser aproximadamente igual à DTG referente ao espectro da amostra. Matematicamente, tem-se:

$\chi^{2}=\sum_{i=1}^{j}\left(\tau_{\text {alvo }}\left(\lambda_{i}\right)-\tau_{\text {teórico }}\left(\lambda_{i}\right)\right)^{2}$

Sendo:

$\tau_{\text {teórico }}(\lambda)$

$=\frac{\pi}{4} \int_{0}^{\infty} Q_{\text {ext }}\left(\lambda, D_{p}\right) D_{p}^{2} f\left(D_{p}\right) d D_{p}$

$\tau_{\text {alvo }}(\lambda)=\ln \frac{I_{T}(\lambda)}{I_{0}(\lambda)}$

A Equação 12 representa a diferença quadrática (resíduo) entre o espectro teórico (do conjunto de espectros teste) e do espectro alvo (correspondente ao espectro da amostra). Logo, a proposta desta técnica consiste em determinar qual espectro-teste produzirá o menor resíduo $\chi^{2}$. A DTG correspondente a este espectro será a DTG procurada.

\section{MÉTODOS DE GERAÇÃO E COMPARAÇÃO DE RESULTADOS}

Nesta etapa do trabalho, foram usados apenas valores de $\tau$ gerados numericamente, a partir de funções de DTG especificadas, aplicando-se o modelo óptico de Mie por meio de scripts em MATLAB $^{\circledR}$. Foram criadas distribuições de tamanho de gotas lognormais (distribuições do logaritmo da variável eliminam inconvenientes práticos quando a variável analisada não pode ser negativa, caso das distribuições de tamanho) e monomodais (por serem a curva multidispersa mais simples de se trabalhar, facilitando a análise preliminar dos algoritmos) e os respectivos espectros de extinção artificiais, eliminando, nesta etapa, procedimentos experimentais para obtenção de dados. Em uma etapa futura do trabalho visa-se utilizar dados reais para fins de validação dos algoritmos.

O procedimento adotado para a criação dos dados artificiais e os testes com os algoritmos de inversão consistem de três etapas:

Etapa 1. Construção das distribuições de tamanho de gotas lognormais e monomodais (denominadas como distribuições originais). A função de distribuição adotada é mostrada abaixo:

$$
\begin{aligned}
& n_{N}\left(D_{p}\right) \\
& =\frac{N_{t}}{(2 \pi)^{1 / 2} D_{p} \ln \sigma_{g}} \exp \left(-\frac{\left(\ln D_{p}-\ln D_{p g}\right)^{2}}{2 \ln ^{2} \sigma_{g}}\right)
\end{aligned}
$$

Os valores da Tabela 1 foram utilizados como parâmetros de geração das distribuições.

Tabela 1 - Parâmetros de geração

\begin{tabular}{cc}
\hline $\begin{array}{c}\text { Diâmetro de moda - } \\
D_{p g}(\mathrm{~nm})\end{array}$ & $\begin{array}{c}\text { Desvio padrão } \\
\text { geométrico }-\sigma_{g}\end{array}$ \\
\hline 500 & 1,1 \\
1000 & 1,3 \\
1500 & 1,5 \\
2000 & \\
\hline
\end{tabular}

O parâmetro $N_{t}$, correspondente ao número de partículas por unidade de volume de emulsão, foi fixado arbitrariamente em $8,45 \cdot 10^{6}$ partículas $/ \mathrm{cm}^{3}$.

Etapa 2. Construção dos espectros de extinção a partir das distribuições originais criadas na primeira etapa.

O modelo de espalhamento de Mie foi adotado para efetuar os cálculos ópticos. Scripts auxiliares propostos por Mätzler (2002a, 2002b) para a determinação das 
eficiências e coeficientes de Mie foram utilizados nesta etapa.

Para cada distribuição de tamanhos criada na etapa 1, foram calculados dois diferentes espectros de turbidez. Cada espectro corresponde a uma emulsão diferente (glicerol em água; poliestireno em água). Isso foi feito para verificar a influência do índice de refração no processo de recriação das distribuições realizado na etapa 3.

As curvas de índice de refração (índice de refração vs. comprimento de onda) necessárias para a criação dos espectros foram obtidas a partir de expressões na literatura. A expressão do índice de refração da água $n_{\text {água }}(\lambda, T, p)$ é dado por Thormahlen et al. (1985); a expressão para o índice de refração do glicerol $n_{\text {glicerol }}(\lambda, T, p)$ é dado por Rheims et al. (1997) e o índice de refração do poliestireno $n_{\text {poliestireno }}(\lambda, T, p)$ é dado por Kasarova et al. (2007).

Etapa 3. Recriação das distribuições de tamanho de gotas a partir dos algoritmos propostos na Seção 2.2 utilizando os espectros da etapa anterior.

Utilizaram-se os algoritmos citados anteriormente (Veselovskii, NNLS, PTR e MBD) para a resolução da Equação (2 e consequente criação de distribuições de tamanho de partículas. Essas novas distribuições são denominadas distribuições recriadas.

De posse das distribuições original (etapa 1) e recriada (etapa 3), deve-se estabelecer critérios comparativos para avaliar a eficiência das técnicas utilizadas. Os critérios escolhidos foram os mais tradicionais, que consistem na comparação do valor médio e do desvio-padrão entre a distribuição original e a recriada.

Para o Método de Busca Direta (MBD) as etapas adotadas foram um pouco diferentes. Um espectro de extinção e sua DTG correspondente são adotados como alvo (criados artificialmente). Um conjunto de espectros-teste é criado a partir de alguns valores de $D_{p g}$ e $\sigma_{g}$ maiores e menores do que aqueles correspondentes à amostra alvo. Feito isso, são calculados os resíduos entre os espectros-teste e o espectro alvo.

\section{RESULTADOS E DISCUSSÃO}

A seguir, é mostrado um gráfico comparativo das DTG's recriadas pelo método de Veselovskii.

Figura 4 - Distribuições originais e recriadas utilizando o método de Veselovskii

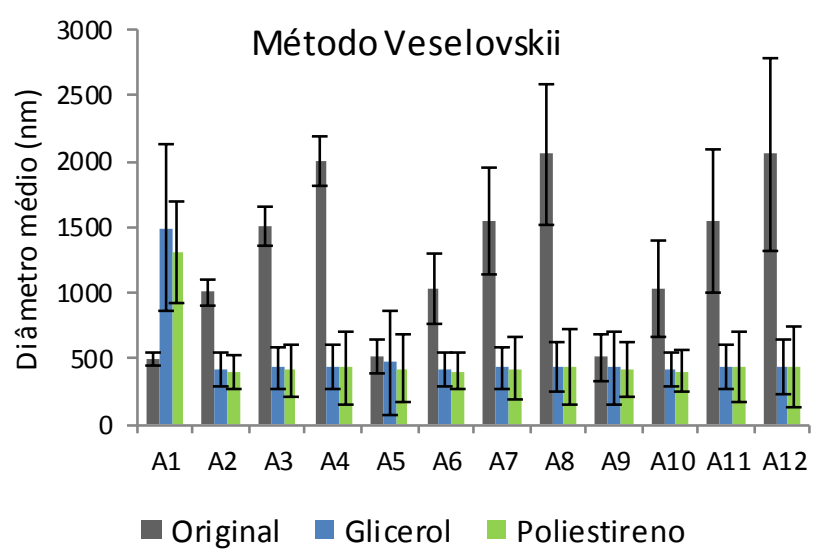

Nota-se pela Figura 4 que o método Veselovskii não forneceu bons resultados, não apresentando sensibilidade em relação aos espectros de extinção, resultando em DTG's próximas, independentemente do espectro de extinção utilizado no processo de inversão.

A Figura 5 contém um comparativo entre as distribuições originais e recriadas através da técnica NNLS. Ela mostra que os diâmetros médios das DTG's recriadas, em que o óleo da emulsão é o poliestireno, se aproximaram significativamente das DTG's originais. No entanto, as DTG's recriadas a partir dos espectros das emulsões contendo glicerol apresentaram grande discrepância em relação às DTG's originais, comparando-se os diâmetros médios. 
Figura 5 - Distribuições originais e recriadas utilizando a técnica NNLS

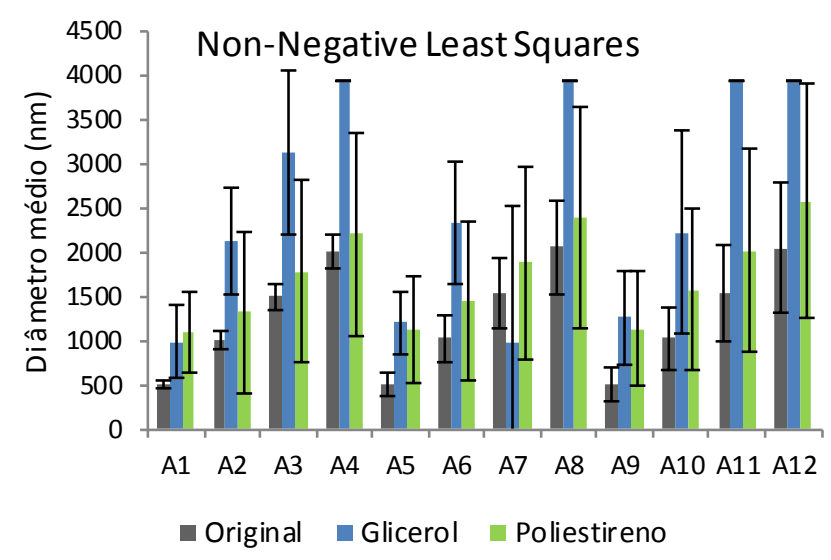

As barras de erro, correspondentes ao desvio padrão das distribuições, mostram que tanto as DTG's recriadas a partir dos espectros das emulsões contendo glicerol, quanto as que contêm poliestireno, estão mais dispersas do que as DTG's originais.

Figura 6 - Distribuições recriadas utilizando a técnica NNLS (Distribuição original: $D_{p g}=$ $500 \mathrm{~nm}$ e $\sigma_{g}=1,3$ )

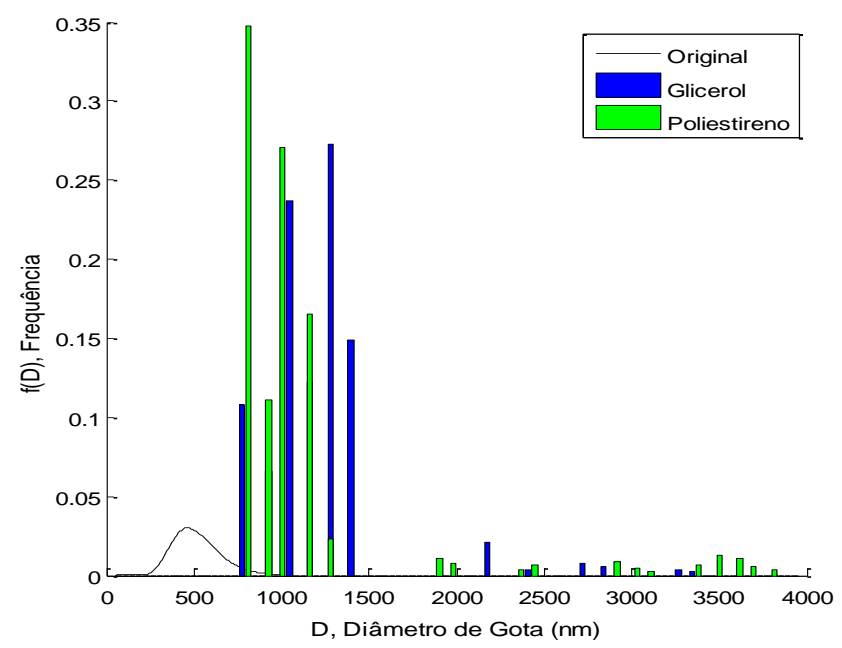

A Figura 6 evidencia a forte dispersão das distribuições recriadas, distantes da distribuição alvo. Esse comportamento pode ser explicado em partes pela restrição de não negatividade. Apesar de forçar a não produção de resultados sem significado físico
- frequências de tamanho de classe com valores negativos - resulta em valores de resíduo maiores no processo de minimização comparados a outras técnicas convencionais. Resíduos maiores contribuem fortemente para essas diferenças tão significativas entre as distribuições originais e recriadas.

A seguir, a Figura 7 mostra o comparativo entre as distribuições originais $\mathrm{e}$ recriadas através da técnica PTR.

Figura 7 - Distribuições originais e recriadas utilizando a técnica PTR

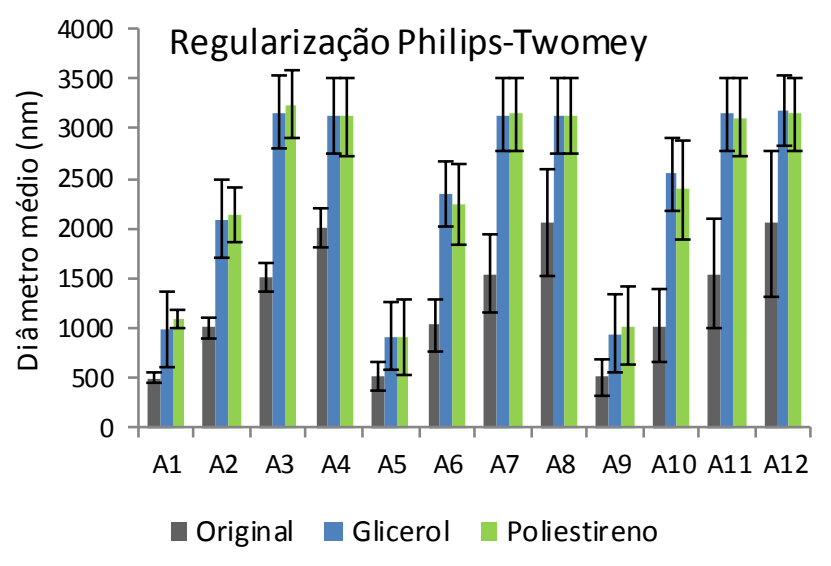

A Figura 7 mostra que o algoritmo PTR não foi sensível aos espectros de extinção correspondentes às DTG's com diâmetro médio superior a 1500nm (A3, A4, A7, A8, A11 e A12), recriando DTG's praticamente iguais em todos esses casos.

O método PTR, no entanto, apresentou melhores resultados para os casos em que o diâmetro médio das DTG's originais era inferior ou igual a $1000 \mathrm{~nm}$. As DTG's recriadas (independentemente de a emulsão conter glicerol ou poliestireno) apresentaram um diâmetro médio cerca de duas vezes maior do que as respectivas DTG's originais. Porém, diferentemente do método NNLS, as distribuições assumiram aspecto monomodal. Neste caso, o cálculo de um parâmetro de ajuste poderia ser uma alternativa para minimizar as discrepâncias entre as DTG's originais e recriadas. 
A Figura 8 mostra que, apesar do deslocamento das DTG's recriadas para diâmetros maiores comparadas à DTG original, a forma monomodal foi mantida.

Figura 8 - Distribuições recriadas utilizando a técnica PTR (Distribuição original: $D_{p g}=500 \mathrm{~nm}$ e $\left.\sigma_{g}=1,3\right)$

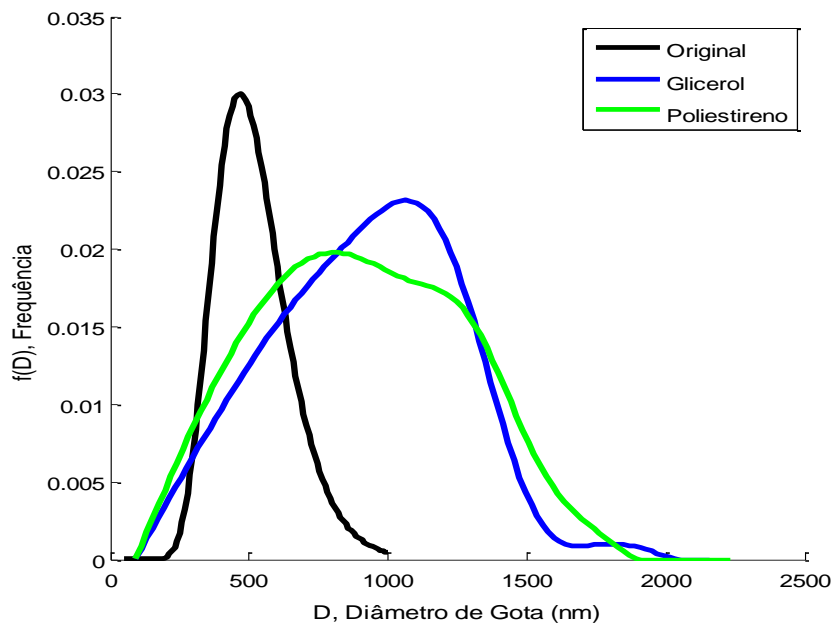

É possível notar também que o método PTR forneceu distribuições com desvios padrões próximos, seja qual fosse o espectro lido pelo algoritmo, como mostra a Figura 7.

Os resultados anteriores mostram que o problema de inversão dificulta sensivelmente uma boa caracterização das distribuições a partir de seus espectros de extinção. Dessa maneira, os próximos resultados (ainda preliminares) correspondem a uma técnica que não utiliza inversão, mas uma comparação de espectros, que é o Método de Busca Direta.

As figuras apresentadas a seguir mostram dois gráficos em cada uma delas: à direita, os espectros "alvo", "calculado" e "esperado"; à esquerda, as DTG's "alvo", "calculada" e "esperada". As curvas "alvo" correspondem à amostra que foi medida experimentalmente (nos casos a seguir, entretanto, trata-se de valores gerados numericamente, como explicado anteriormente, que serão validados experimentalmente em uma etapa futura). As curvas "calculadas" são aquelas que o algoritmo determinou como sendo as mais próximas das curvas "alvo", ou seja, o espectro "calculado" é o que apresentou a menor distância quadrática em relação ao espectro "alvo". As curvas "esperadas" são aquelas cuja DTG é, de fato, a mais próxima da "alvo". $\mathrm{Na}$ prática, essas curvas "esperadas" não são conhecidas, mas como neste trabalho são utilizados valores gerados numericamente, a distribuição alvo é conhecida, e é possível prever qual é a distribuição esperada dentre aquelas presentes no conjunto 'teste". Dessa maneira, é possível dizer se o método fornece ou não a melhor distribuição para cada caso estudado.

A Figura 9 mostra um caso em que as curvas "calculadas" e "esperadas" são coincidentes. Ou seja, o resultado fornecido pela técnica foi o melhor possível.

Figura 9 - Distribuição recriada utilizando a técnica MBD (Distribuição alvo: $D_{p g}=1000 \mathrm{~nm}$ e $\left.\sigma_{g}=1,1\right)$
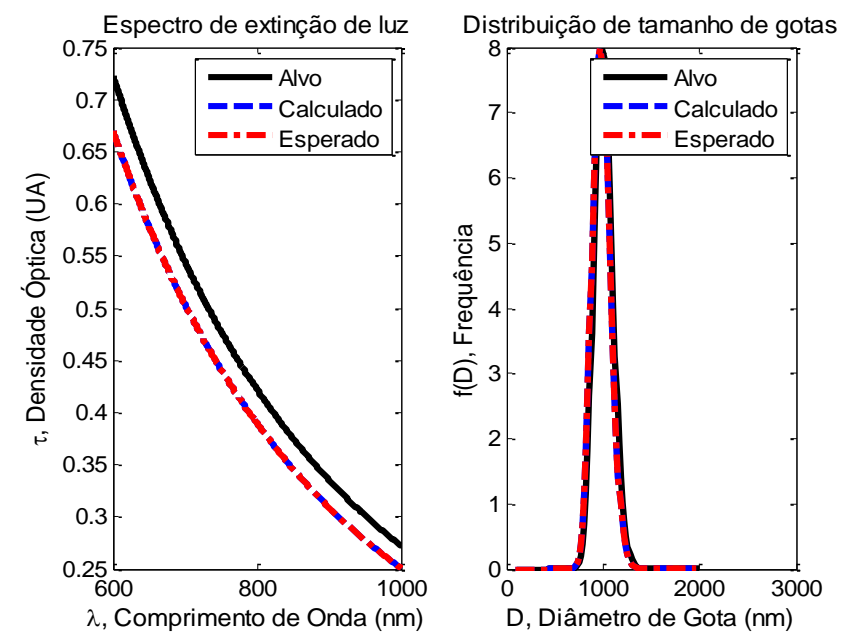

No entanto, nem sempre espectros de extinção próximos resultam em DTG's próximas. Isso evidencia a dificuldade em obter as distribuições de tamanho a partir de técnicas espectroscópicas. A Figura 10 ilustra um caso em que espectros próximos não levam a distribuições próximas. 
Figura 10 - Distribuição recriada utilizando a técnica MBD (Distribuição alvo: $D_{p g}=500 \mathrm{~nm}$ e $\left.\sigma_{g}=1,3\right)$
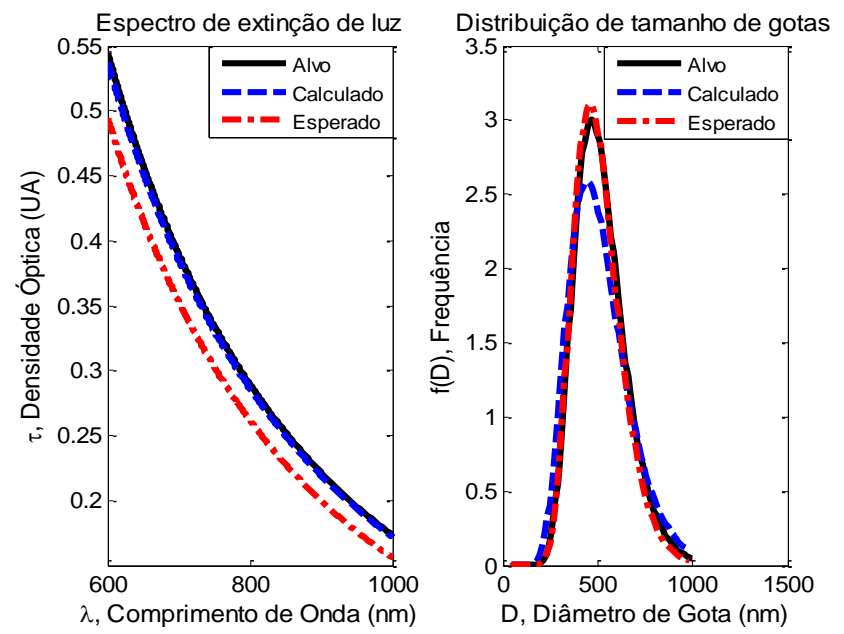

Nota-se na Figura 10 que as curvas "calculada" e "esperada" não são coincidentes. Isso é consequência do fato de que espectros próximos não resultam necessariamente em DTG's próximas. Neste exemplo, o espectro mais próximo do alvo (espectro calculado) quase coincide com o espectro alvo, enquanto o espectro esperado é claramente mais distante. As DTG's, no entanto, se comportam de maneira oposta, sendo a esperada quase coincidente com a DTG alvo.

É importante ressaltar que, apesar do Método de Busca Direta nem sempre fornecer a melhor distribuição dentre as DTG's possíveis, ainda se obtém uma distribuição próxima da desejada.

\section{CONCLUSÃO}

Os algoritmos que utilizam técnicas de inversão (Veselovskii, NNLS e PTR) mostraram em seus resultados a dificuldade em conseguir extrair dos espectros de extinção informações suficientes para se obterem DTG's de boa qualidade.

O método proposto por Veselovskii não se mostrou interessante devido à sensibilidade quase nula em relação às informações artificiais de turbidez, resultando em DTG's praticamente idênticas para todos os espectros testados. A técnica NNLS se mostrou mais eficiente, porém as fortes dispersões das distribuições recriadas prejudicaram a qualidade das mesmas. A técnica PTR, por sua vez, conseguiu reproduzir distribuições monomodais, como era esperado, e estas distribuições recriadas se apresentaram deslocadas para diâmetros de partículas maiores. A inserção de um parâmetro de correção pode ser uma alternativa para solucionar tal problema. Para distribuições contendo partículas maiores $\left(D_{p g} \geq\right.$ $1500 \mathrm{~nm})$, a técnica PTR não se mostrou eficiente, não apresentando sensibilidade suficiente em relação aos espectros de extinção.

O Método de Busca Direta, único dos métodos testados que não utilizou uma técnica de inversão para recriar as DTG's através de espectros, se mostrou bastante promissor. A partir dele foi possível construir DTG's próximas das DTG's esperadas.

\section{NOMENCLATURA}

A Matriz óptica

$B_{j} \quad$ Função $B$-spline

$C_{j} \quad$ Função peso

$D_{p} \quad$ Diâmetro de partícula, $\mathrm{nm}$

$D_{p g} \quad$ Diâmetro mediano das partículas, $\mathrm{nm}$

$f \quad$ Distribuição de tamanhos de partículas

$H \quad$ Matriz de suavização

$K \quad$ Função Kernel

$n_{N} \quad$ Função densidade de distribuição

$N_{t} \quad$ Número total de partículas por unidade de volume, partículas $/ \mathrm{cm}^{3}$

$Q_{\text {ext }}$ Eficiência de extinção

$r \quad$ Raio de partícula, $\mathrm{nm}$

$\alpha \quad$ Raio de partícula adimensional

$\gamma \quad$ Parâmetro de regularização

$\varepsilon \quad$ Erro

$\lambda$ Comprimento de onda, $\mathrm{nm}$

$\sigma_{g} \quad$ Desvio padrão geométrico 
$\begin{array}{ll}\tau & \text { Turbidez/extinção, UA } \\ \chi & \text { Resíduo }\end{array}$

\section{REFERÊNCIAS}

BOHREN, C. F.; HUFFMAN, D. R. Absorption and Scattering of Light by Small Particles. 1. ed. New York: John Wiley \& Sons, Inc, 1983.

ELICABE, G. E.; GARCIA-RUBIO, L. H. Latex particle size distribution from turbidimetry using a combination of regularization techniques and generalized cross validation. Advances in chemistry series, v. 227, p. 83-104, 1990.

\section{FRIEDLANDER, S. K. Smoke Dust and} Haze: Fundamentals of Aerosol Dynamics. 2. ed. New York: Oxford University Press, 2000 .

GREEN, P. J.; SILVERMAN, B. W. Nonparametric Regression and Generalized Linear Models: A Roughness Penalty Approach. 1. ed. London: Chapman \& Hall, 1994. v. 58

KASAROVA, S. N. et al. Analysis of the dispersion of optical plastic materials.

Optical Materials, v. 29, p. 1481-1490, 2007.

KHATIB, M. Contemporary Issues in Wireless Communications. [s.1.] InTech, 2014.

LAWSON, C. L.; HANSON, R. J. Solving

Least Square Problems. Reprint ed.Philadelphia: SIAM: Society for Industrial and Applied Mathematics, 1995.

MÄTZLER, C. MATLAB Functions for Mie Scattering and Absorption Version 2. Bern: [s.n.].
MÄTZLER, C. MATLAB Functions for Mie Scattering and Absorption. Bern: [s.n.].

RHEIMS, J.; KÖSER, J.; WRIEDT, T.

Refractive-index measurements in the near-IR using an Abbe refractometer. Measurement Science and Technology, v. 8, n. 6, p. 601605, 1 jun. 1997.

SEINFELD, J.; PANDIS, S. Atmospheric chemistry and physics: from air pollution to climate change. $2^{\mathrm{a}}$. ed. New Jersey: John Wiley \& Sons, Inc, 2006.

\section{TARANTOLA, A. Inverse Problem Theory} and Methods for Model Parameter Estimation. 1. ed. Philadelphia: SIAM:

Society for Industrial and Applied Mathematics, 2004.

THORMAHLEN, I.; STRAUB, J.; GRIGULL, U. Refractive index of water and its dependence on wavelength, temperature and density. Journal of Physical and Chemical Reference Data, v. 14, n. 4, p. 933-945, 1985.

TWOMEY, S. Introduction to the Mathematics of Inversion in Remote Sensing and Indirect Measurements. 1. ed. Mineola, New York: Elsevier, 1977.

VAN DE HULST, H. C. Light Scattering by Small Particles. Reprint ed.New York: Dover Publications, 1981.

VESELOVSKII, I. et al. Inversion with regularization for the retrieval of tropospheric aerosol parameters from multiwavelength lidar sounding. Applied optics, v. 41, n. 18, p. 3685-99, 20 jun. 2002.

\section{AGRADECIMENTOS}

Os autores agradecem à CAPES, CNPq e BRAGECRIM pelo apoio a esta pesquisa. 